\title{
ON A VARIATIONAL PROBLEM ARISING IN CRYSTALLOGRAPHY
}

\author{
AleXANDER J. ZASLAVSKI ${ }^{1}$
}

\begin{abstract}
We study a variational problem which was introduced by Hannon, Marcus and Mizel [ESAIM: COCV 9 (2003) 145-149] to describe step-terraces on surfaces of so-called "unorthodox" crystals. We show that there is no nondegenerate intervals on which the absolute value of a minimizer is $\pi / 2$ identically.
\end{abstract}

Mathematics Subject Classification. 49J99, 74E15.

Received October 28, 2004. Revised June 26, 2005.

\section{IntRoduction}

For the understanding of crystalline growth processes, the form of step-terraces on the crystalline surface plays an important role [5]. The edges of these steps usually form oscillations in space that become larger when the equilibrium temperature rises. This behavior is called "orthodox" and had been explained by Herring, Mullins and others (see e.g. [6]) by thermodynamical effects. The classical model is given by

$$
J_{1}(y)=\int_{0}^{S} \beta(\theta) \mathrm{d} s
$$

where $s$ is arclength and $y$ is a function defined on a fixed interval $[0, L]$ whose graph is the locus under consideration:

$$
y \in W^{1,1}(0, L), \theta=\arctan y^{\prime} \in[-\pi / 2, \pi / 2],
$$

while $\beta$ is a positive $\pi$-periodic function which satisfies certain properties. Minimization of $J_{1}$ subject to appropriate boundary data is a parametric variational problem. It is closely related to the variational problem defining the Wulff crystal shape as that shape for a domain of prescribed area such that the boundary integral with respect to arclength involving the integrand in $J_{1}$ [referred to as the surface tension] attains its minimum value $[1,2]$. Recently crystals have been studied which are "unorthodox" in the sense that lower temperatures lead to larger oscillations and the step profile takes a saw-tooth structure for low temperatures and not a straight line as the classical theory would predict [3]. To describe this situation, Hannon, Marcus and Mizel [4] suggested a refined model which will be stated below.

Keywords and phrases. Minimizer, surfaces of crystals, unorthodox crystal, variational problem.

1 Department of Mathematics, Technion-Israel Institute of Technology, 32000, Haifa, Israel; ajzasltx.technion.ac.il

(c) EDP Sciences, SMAI 2007 
Suppose that a function $\beta \in C(R)$ satisfies the following assumption:

(A)

$$
\begin{gathered}
\beta(t)>0 \text { for all } t \in R, \\
\beta(t)=\beta(-t) \text { for all } t \in R, \\
\beta(\pi / 2) \leq \beta(t) \leq \beta(0) \text { for all } t \in R, \\
\beta(t+\pi)=\beta(t) \text { for all } t \in R .
\end{gathered}
$$

Let $L>0, \rho>0, \sigma>0$. We study the following variational problem introduced in [4]:

$$
\begin{gathered}
J_{\rho \sigma}^{L}(\theta, y):=\int_{0}^{S}\left[\rho\left(\theta^{\prime}(s)\right)^{2}+\beta(\theta(s))+\sigma y(s)^{2}\right] \mathrm{d} s \rightarrow \inf , \\
y(s)=y(0)+\int_{0}^{s} \sin (\theta(\tau)) \mathrm{d} \tau, s \in[0, S],
\end{gathered}
$$

where $S \geq L, \theta \in W^{1,2}(0, S)$ is subject to the following constraints:

$$
\begin{gathered}
\theta(\tau) \in[-\pi / 2, \pi / 2], \tau \in[0, S], \int_{0}^{S} \cos (\theta(\tau)) \mathrm{d} \tau=L, \\
\int_{0}^{S} \sin (\theta(\tau)) \mathrm{d} \tau=0 .
\end{gathered}
$$

Here $\theta$ describes the angle of the step profile relative to a straight line profile. The first constraint in (1.7) expresses the condition that the curve

$$
(x(s), y(s)):=\left(\int_{0}^{s} \cos (\theta(\tau)) \mathrm{d} \tau, y(0)+\int_{0}^{s} \sin (\theta(\tau)) \mathrm{d} \tau\right)
$$

does not "reverse", the second is the condition that the $x$-interval is $[0, L]$, while the third condition in (1.7) is the condition that $y(0)=y(S)$.

It was shown in [4] that problem (1.5)-(1.7) has a solution. Actually in [4] it was assumed that $\beta \in C^{2}(R)$ and that $\beta(0)+\beta^{\prime \prime}(0)<0$ but the existence result of [4] holds without these two additional assumptions and with the same proof. Hannon, Marcus and Mizel [4] noted that their theorem does not exclude the possibility that a minimizer $(S, \theta, y)$ satisfies $|\theta|=\pi / 2$ on one or more nondegenerate intervals. If this occurs, then the locus of the curve $s \rightarrow(x(s), y(s)), s \in[0, S]$ is not the graph of a function defined on $[0, L]$. This fact leads to difficulties in calculating a solution.

Our main result stated below establishes that if a parameter $\sigma$ is small enough, then the locus of the curve $s \rightarrow(x(s), y(s)), s \in[0, S]$ associated with a minimizer $(S, \theta, y)$ is necessarily a graph of a function defined on $[0, L]$. It should be mentioned that the smallness of $\sigma$ is a natural assumption for the model.

Theorem 1.1. Let $\rho_{1}, L_{1}>0$. Then there is $\sigma_{1}>0$ such that for each $\rho \geq \rho_{1}$, each $L \in\left(0, L_{1}\right]$ and each $\sigma \in\left(0, \sigma_{1}\right]$ the following assertion holds:

Assume that $(S, \theta, y)$ is a solution of the problem (1.5)-(1.7). Then there is no interval $[a, b] \subset[0, S]$ such that $a<b$ and $|\theta(t)|=\pi / 2$ for all $t \in[a, b]$.

The proof of Theorem 1.1 is long and technical. It is based on a number of auxiliary results. Here we explain the main ideas of the proof.

In the proof of Theorem 1.1 we use two procedures applied to triples $(S, \theta, y)$ : a reduction of a triplet and a restriction of a triplet.

Let $S \geq L, \theta \in W^{1,2}(0, S)$ and $y:[0, S] \rightarrow R$ satisfy (1.6) and (1.7). 
Assume that $t_{0} \in[0, S]$ and $\delta>0$. An extension of the triplet $(S, \theta, y)$ is a triplet $(\tilde{S}, \tilde{\theta}, \tilde{y})$ defined by

$$
\begin{gathered}
\tilde{S}=S+\delta, \tilde{\theta}(t)=\theta(t), t \in\left[0, t_{0}\right], \tilde{\theta}(t)=\theta\left(t_{0}\right), t \in\left(t_{0}, t_{0}+\delta\right], \\
\tilde{\theta}(t)=\theta(t-\delta), t \in\left(t_{0}+\delta, \tilde{S}\right], \tilde{y}(\tau)=y(0)+\int_{0}^{\tau} \sin (\tilde{\theta}(t)) \mathrm{d} t, \tau \in[0, \tilde{S}] .
\end{gathered}
$$

Let us now describe a reduction of the triplet $(S, \theta, y)$. Assume that $\Delta=[a, b] \subset[0, S]$ and that one of the following cases holds:

(1) $a=0$; (2) $b=S$; (3) $0<a, b<S$ and $\theta(a)=\theta(b)$.

Put $\tilde{S}=S-b+a$. In the case $(1)$ set $\tilde{\theta}(t)=\theta(t+b-a), t \in[0, \tilde{S}]$, in the case $(2)$ set $\tilde{\theta}(t)=\theta(t), t \in[0, \tilde{S}]$ and in the case $(3)$ set $\tilde{\theta}(t)=\theta(t), t \in[0, a], \tilde{\theta}(t)=\theta(t+b-a), t \in(a, \tilde{S}]$. Finally we define

$$
\tilde{y}(\tau)=y(0)+\int_{0}^{\tau} \sin (\tilde{\theta}(t)) \mathrm{d} t, \tau \in[0, \tilde{S}]
$$

The triplet $(\tilde{S}, \tilde{\theta}, \tilde{y})$ is called a reduction of the triplet $(S, \theta, y)$.

We prove Theorem 1.1 by negation. We assume that a triplet $\left(S_{0}, \theta_{0}, y_{0}\right)$ is a solution of the variational problem and that $\left|\theta_{0}(t)\right|=\pi / 2$ for all $t$ belonging to a subinterval of $\left[0, S_{0}\right]$ with a positive length. Using the extension of triples and the reduction of triples we will construct a new admissible triplet $\left(S_{1}, \theta_{1}, y_{1}\right)$ such that $J_{\rho \sigma}^{L}\left(\theta_{1}, y_{1}\right)<J_{\rho \sigma}^{L}\left(\theta_{0}, y_{0}\right)$. In order to meet this goal we will choose a small positive constant $r_{0}$ and consider separately two cases:

$$
\inf \left\{\theta_{0}(t): t \in\left[0, S_{0}\right]\right\} \leq-\pi / 2+r_{0} \text { and } \inf \left\{\theta_{0}(t): t \in\left[0, S_{0}\right]\right\}>-\pi / 2+r_{0}
$$

\section{Auxiliary Results}

For each function $f: X \rightarrow R$, where $X$ is nonempty, set $\inf (f)=\inf \{f(x): x \in X\}$. Denote by meas $(E)$ the Lebesgue measure of a Lebesgue measurable set $E \subset R$.

Lemma 2.1. Let $L, \rho, \sigma>0$. Then $\inf \left(J_{\rho \sigma}^{L}\right) \leq L \beta(0)$.

Proof. Set $S=L, \theta(t)=0, t \in[0, S]$ and $y(t)=0, t \in[0, S]$. Clearly $(S, \theta, y)$ satisfies (1.6) and (1.7). Then $\inf \left(J_{\rho \sigma}^{L}\right) \leq J_{\rho \sigma}^{L}(\theta, y)=L \beta(0)$. Lemma 2.1 is proved.

Lemma 2.2. Let $L, \rho, \sigma>0$ and let $S \geq L, \theta \in W^{1,2}(0, S), y:[0, S] \rightarrow R$ satisfy (1.6) and (1.7). Then

$$
S \leq\left[L \beta(0)+J_{\rho \sigma}^{L}(\theta, y)-\inf \left(J_{\rho \sigma}^{L}\right)\right](\beta(\pi / 2))^{-1} .
$$

Proof. It follows from (1.5), (1.3) and Lemma 2.1 that

$$
\begin{aligned}
S \beta(\pi / 2) & \leq J_{\rho \sigma}^{L}(\theta, y)=\inf \left(J_{\rho \sigma}^{L}\right)+\left[J_{\rho \sigma}^{L}(\theta, y)-\inf \left(J_{\rho \sigma}^{L}\right)\right] \\
& \leq L \beta(0)+\left[J_{\rho \sigma}^{L}(\theta, y)-\inf \left(J_{\rho \sigma}^{L}\right)\right] .
\end{aligned}
$$

This inequality implies (2.1).

Corollary 2.1. Let $L, \rho, \sigma>0$ and let $(S, \theta, y)$ be a solution of the problem $(1.5)-(1.7)$. Then $S \leq L \beta(0)(\beta(\pi / 2))^{-1}$.

Corollary 2.2. Let $L, \rho, \sigma>0$ and let $S \geq L, \theta \in W^{1,2}(0, S), y:[0, S]$ satisfy (1.6) and (1.7). Assume that $J_{\rho \sigma}^{L}(\theta, y) \leq \inf \left(J_{\rho \sigma}^{L}\right)+L \beta(0)$. Then

$$
S \leq 2 L \beta(0)(\beta(\pi / 2))^{-1}
$$


Lemma 2.3. Let $L, \rho, \sigma>0$ and let $S \geq L, \theta \in W^{1,2}(0, S), y:[0, S] \rightarrow R$ satisfy (1.6) and (1.7). Assume that

$$
J_{\rho \sigma}^{L}(\theta, y) \leq \inf \left(J_{\rho \sigma}^{L}\right)+\min \left\{L \beta(0), \sigma L^{3}\right\}
$$

Then

$$
|y(t)| \leq 8 L \beta(0)(\beta(\pi / 2))^{-1} \text { for all } t \in[0, S] .
$$

Proof. By (2.2) and Corollary 2.2

$$
S \leq 2 L \beta(0)(\beta(\pi / 2))^{-1} .
$$

Relations (1.6) and (2.4) imply that for each $t \in[0, S]$

$$
|y(t)-y(0)| \leq t \leq S \leq 2 L \beta(0)(\beta(\pi / 2))^{-1} .
$$

Set

$$
S_{1}=S, \theta_{1}=\theta, y_{1}(\tau)=\int_{0}^{\tau} \sin (\theta(t)) \mathrm{d} t, \tau \in[0, S]
$$

Clearly

$$
\left|y_{1}(t)\right| \leq t \text { for all } t \in[0, S]
$$

and (1.6), (1.7) hold with $(S, \theta, y)=\left(S_{1}, \theta_{1}, y_{1}\right)$. It follows from (2.2), (2.6) and (1.5) that

$$
J_{\rho \sigma}^{L}(\theta, y) \leq J_{\rho \sigma}^{L}\left(\theta_{1}, y_{1}\right)+\sigma L^{3}=J_{\rho \sigma}^{L}(\theta, y)+\sigma\left[\int_{0}^{S}\left(y_{1}(t)\right)^{2} \mathrm{~d} t-\int_{0}^{S}(y(t))^{2} \mathrm{~d} t\right]+\sigma L^{3}
$$

and

$$
\int_{0}^{S}(y(t))^{2} \mathrm{~d} t \leq \int_{0}^{S}\left(y_{1}(t)\right)^{2} \mathrm{~d} t+L^{3}
$$

Combined with (2.7) this implies that

$$
\int_{0}^{S}(y(t))^{2} \mathrm{~d} t \leq L^{3}+S^{3} \leq 2 S^{3}
$$

We show that $|y(0)| \leq 3 S$. Let us assume the converse. Then $|y(0)|>3 S$ and by $(2.5)|y(t)|>2 S$ for all $t \in$ $[0, S]$. This inequality implies that $\int_{0}^{S}(y(t))^{2} \mathrm{~d} t \geq 4 S^{3}$. This inequality contradicts (2.8). The contradiction we have reached proves that $|y(0)| \leq 3 S$. Combined with (2.5) and (2.4) this inequality implies that for all $t \in[0, S]$

$$
|y(t)| \leq|y(0)|+S \leq 4 S \leq 8 L \beta(0)(\beta(\pi / 2))^{-1} .
$$

Lemma 2.3 is proved.

Lemma 2.4. Let $L, \rho, \sigma>0, S \geq L, \theta \in W^{1,2}(0, S), y:[0, S] \rightarrow R$ satisfy (1.6) and (1.7). Suppose that $(S, \theta, y)$ is a solution of the problem $(1.5)-(1.7)$. Then

$$
\int_{0}^{S}\left(\theta^{\prime}(t)\right)^{2} \mathrm{~d} t \leq \rho^{-1} L \beta(0)
$$

and for each $t_{1}, t_{2} \in[0, S]$ satisfying $t_{1}<t_{2}$ the following inequality holds:

$$
\left|\theta\left(t_{2}\right)-\theta\left(t_{1}\right)\right| \leq\left(\rho^{-1} L \beta(0)\left(t_{2}-t_{1}\right)\right)^{1 / 2} .
$$


Proof. By Corollary $2.1 L \leq S \leq L \beta(0) \beta(\pi / 2)^{-1}$. It follows from (1.5) and Lemma 2.1 that

$$
\int_{0}^{S}\left(\theta^{\prime}(t)\right)^{2} \mathrm{~d} t \leq \rho^{-1} J_{\rho \sigma}^{L}(\theta, y)=\rho^{-1} \inf \left(J_{\rho \sigma}^{L}\right) \leq \rho^{-1} L \beta(0) .
$$

Inequality (2.10) follows from (2.9) by the Cauchy-Schwarz inequality.

Lemma 2.5. Let $L_{1}>0, \rho>0$ and let a positive number $\gamma$ satisfy

$$
\gamma \leq \arcsin \left(2^{-1}(\beta(0))^{-1} \beta(\pi / 2) \min \left\{1,\left(\pi^{2} / 16\right) \rho L_{1}^{-2}(\beta(0))^{-1}\right\}\right) .
$$

Suppose that $\sigma>0, L \in\left(0, L_{1}\right]$ and that $S \geq L, \theta \in W^{1,2}(0, S)$ and $y:[0, S] \rightarrow R$ are a solution of the problem (1.5)-(1.7) such that

$$
\max \{\theta(t): t \in[0, S]\}=\pi / 2
$$

Then

$$
\min \{\theta(t): t \in[0, S]\} \leq-\gamma .
$$

Proof. By (2.12) there is $t_{0} \in[0, S]$ such that

$$
\theta\left(t_{0}\right)=\pi / 2
$$

Set

$$
E=[0, S] \cap\left[t_{0}-(\pi / 4)^{2} \rho L^{-1}(\beta(0))^{-1}, t_{0}+(\pi / 4)^{2} \rho L^{-1}(\beta(0))^{-1}\right] .
$$

Assume that $t \in E$. By (2.15) and Lemma 2.4

$$
\left|\theta(t)-\theta\left(t_{0}\right)\right| \leq\left(\rho^{-1} L \beta(0)\left|t-t_{0}\right|\right)^{1 / 2} \leq \pi / 4 .
$$

Combined with (2.14) this inequality implies that $\theta(t) \geq \pi / 4$. Thus we have shown that

$$
\theta(t) \geq \pi / 4 \text { for all } t \in E .
$$

Clearly

$$
\operatorname{meas}(E) \geq \min \left\{(\pi / 4)^{2} \rho L^{-1} \beta(0)^{-1}, S\right\} .
$$

Relations (2.15), (2.16), (2.17) and (1.7) imply that

$$
\int_{E} \sin (\theta(t)) \mathrm{d} t \geq \sin (\pi / 4) \text { meas }(E) \geq 2^{-1} \min \left\{(\pi / 4)^{2} \rho L^{-1}(\beta(0))^{-1}, S\right\} .
$$

By (2.18) and (1.7)

$$
\int_{[0, S \backslash \backslash E} \sin (\theta(t)) \mathrm{d} t=-\int_{E} \sin (\theta(t)) \mathrm{d} t \leq-2^{-1} \min \left\{(\pi / 4)^{2} \rho L^{-1}(\beta(0))^{-1}, S\right\} .
$$

Since $\inf (\theta) \leq 0$ (see (1.7)) the relation (2.19) implies that

$$
\begin{aligned}
-2^{-1} \min \left\{(\pi / 4)^{2} \rho L^{-1}(\beta(0))^{-1}, S\right\} & \geq \int_{[0, S] \backslash E} \sin (\theta(t)) \mathrm{d} t \\
& \geq \sin (\inf (\theta)) \operatorname{meas}([0, S] \backslash E) \geq \sin (\inf (\theta)) S .
\end{aligned}
$$


It follows from (2.20), Corollary 2.1 and the inequality $L \leq L_{1}$ that

$$
\begin{aligned}
\sin (\inf (\theta)) & \leq-2^{-1} \min \left\{(\pi / 4)^{2} \rho L^{-1}(\beta(0))^{-1}, L\right\} L^{-1}(\beta(0))^{-1} \beta(\pi / 2) \\
& \leq-2^{-1}(\beta(0))^{-1} \beta(\pi / 2) \min \left\{1,(\pi / 4)^{2} \rho L_{1}^{-2}(\beta(0))^{-1}\right\}
\end{aligned}
$$

and in view of (2.11)

$$
\inf (\theta) \leq-\arcsin \left(2^{-1}(\beta(0))^{-1} \beta(\pi / 2) \min \left\{1,(\pi / 4)^{2} \rho L_{1}^{-2}(\beta(0))^{-1}\right\} \leq-\gamma .\right.
$$

This completes the proof of Lemma 2.5.

It is easy to see that the following lemma holds.

Lemma 2.6. Let $S>0, \theta:[0, S] \rightarrow R$ be a continuous function, $M>0, \delta \in(0, S)$ and let $y:[0, S] \rightarrow R$ satisfy

$$
y(\tau)=y(0)+\int_{0}^{\tau} \sin (\theta(t)) \mathrm{d} t, \tau \in[0, S]
$$

and

$$
|y(\tau)| \leq M \text { for all } \tau \in[0, S]
$$

Suppose that

$$
\begin{gathered}
\tilde{S}=S-\delta, \tilde{\theta}(t)=\theta(t+\delta), t \in[0, \tilde{S}], \\
\tilde{y}(\tau)=y(0)+\int_{0}^{\tau} \sin (\tilde{\theta}(t)) \mathrm{d} t, \tau \in[0, \tilde{S}] .
\end{gathered}
$$

Then

$$
|\tilde{y}(t)| \leq M+\delta \text { for all } \tau \in[0, \tilde{S}]
$$

and

$$
\left|\int_{0}^{S}(y(t))^{2} \mathrm{~d} t-\int_{0}^{\tilde{S}}(\tilde{y}(t))^{2} \mathrm{~d} t\right| \leq M^{2} \delta+\delta S(2 M+\delta) .
$$

Lemma 2.7. Let $S>0, \theta:[0, S] \rightarrow R$ be a continuous function, $M>0,0<a<b<S$ and let $y:[0, S] \rightarrow R$ satisfy (2.21) and (2.22). Suppose that

$$
\begin{gathered}
\theta(a)=\theta(b), \\
\tilde{S}=S-b+a, \\
\tilde{\theta}(t)=\theta(t), t \in[0, a], \tilde{\theta}(t)=\theta(t+b-a), t \in(a, \tilde{S}], \\
\tilde{y}(\tau)=y(0)+\int_{0}^{\tau} \sin (\tilde{\theta}(t)) \mathrm{d} t, \tau \in[0, \tilde{S}] .
\end{gathered}
$$

Then

$$
|\tilde{y}(\tau)| \leq M+b-a \text { for all } \tau \in[0, \tilde{S}]
$$

and

$$
\left|\int_{0}^{S}(y(t))^{2}-\int_{0}^{\tilde{S}}(\tilde{y}(t))^{2} \mathrm{~d} t\right| \leq(b-a) M^{2}+(b-a)(2 M+b-a) S .
$$


Proof. Relations (2.21), (2.29), (2.30) imply that

$$
\tilde{y}(t)=y(t), t \in[0, a] .
$$

Assume that $\tau \in(a, \tilde{S})$. It follows from (2.30), (2.29) and (2.21) that

$$
\begin{aligned}
\tilde{y}(\tau) & =y(0)+\int_{0}^{a} \sin (\theta(t)) \mathrm{d} t+\int_{a}^{\tau} \sin (\theta(t+b-a)) \mathrm{d} t \\
& =y(0)+\int_{0}^{\tau+b-a} \sin (\theta(t)) \mathrm{d} t-\int_{a}^{b} \sin (\theta(t)) \mathrm{d} t \\
& =y(\tau+b-a)-\int_{a}^{b} \sin (\theta(t)) \mathrm{d} t .
\end{aligned}
$$

This equality implies that

$$
|\tilde{y}(\tau)-y(\tau+b-a)| \leq b-a \text { for all } \tau \in(a, \tilde{S}) .
$$

Combined with (2.22) the inequality (2.34) implies that

$$
|\tilde{y}(\tau)| \leq M+b-a \text { for all } \tau \in(a, \tilde{S}) .
$$

Relations (2.22), (2.33), (2.35) imply (2.31). It follows from (2.33), (2.22), (2.28), (2.31) that

$$
\begin{gathered}
\left|\int_{0}^{S}(y(t))^{2} \mathrm{~d} t-\int_{0}^{\tilde{S}}(\tilde{y}(t))^{2} \mathrm{~d} t\right| \\
\leq \int_{a}^{b}(y(t))^{2} \mathrm{~d} t+\left|\int_{a}^{\tilde{S}}(y(t+b-a))^{2} \mathrm{~d} t-\int_{a}^{\tilde{S}}(\tilde{y}(t))^{2} \mathrm{~d} t\right| \\
\leq(b-a) M^{2}+\int_{a}^{\tilde{S}}|y(t+b-a)-\tilde{y}(t)|(|y(t+b-a)|+|\tilde{y}(t)|) \mathrm{d} t \\
\leq(b-a) M^{2}+(b-a) S(2 M+b-a) .
\end{gathered}
$$

Thus (2.32) is true and Lemma 2.7 is proved.

The following auxiliary result is proved analogously to Lemma 2.7 .

Lemma 2.8. Let $S>0, \theta:[0, S] \rightarrow R$ be a continuous function, $M>0,0 \leq a \leq S, \delta>0$ and let $y:[0, S] \rightarrow R$ satisfy (2.21) and (2.22). Suppose that

$$
\begin{gathered}
\tilde{S}=S+\delta, \\
\tilde{\theta}(t)=\theta(t), t \in[0, a], \tilde{\theta}(t)=\theta(a), t \in(a, a+\delta], \\
\tilde{\theta}(t)=\theta(t-\delta), t \in(a+\delta, \tilde{S}], \\
\tilde{y}(\tau)=y(0)+\int_{0}^{\tau} \sin (\tilde{\theta}(t)) \mathrm{d} t, \tau \in[0, \tilde{S}] .
\end{gathered}
$$

Then

$$
|\tilde{y}(\tau)| \leq M+\delta \text { for all } \tau \in[0, \tilde{S}]
$$

and

$$
\left|\int_{0}^{S}(y(t))^{2} \mathrm{~d} t-\int_{0}^{\tilde{S}}(\tilde{y}(t))^{2} \mathrm{~d} t\right| \leq \delta(M+\delta)^{2}+\delta S(2 M+\delta) .
$$




\section{A Weakened version of Theorem 1.1}

In this section we establish the following result.

Theorem 3.1. There exists $r_{0} \in(0, \pi / 8)$ such that for each $L_{0}>0$ there is $\sigma_{0}>0$ for which the following assertion holds:

Suppose that $L \in\left(0, L_{0}\right], \rho>0, \sigma \in\left(0, \sigma_{0}\right],(S, \theta, y)$ is a solution of the problem $(1.5)-(1.7)$ and

$$
\left[-\pi / 2+r_{0}, \pi / 2-r_{0}\right] \subset \theta([0, S])
$$

Then there is no interval $[a, b] \subset[0, S]$ such that $a<b$ and $|\theta(t)|=\pi / 2$ for all $t \in[a, b]$.

Proof. Choose a positive number $r_{1}$ such that

$$
r_{1} \in(0, \pi / 16), \beta(0) \cos \left(\pi / 2-r_{1}\right) \leq \beta(\pi / 2) / 16
$$

and choose

Let $L_{0}>0$. Put

$$
r_{0} \in\left(0, r_{1} / 2\right)
$$

and choose a positive number $\sigma_{0}$ such that

$$
\Delta_{0}=16 L_{0} \beta(0)(\beta(\pi / 2))^{-1}
$$

$$
\sigma_{0} \Delta_{0}^{2}<\beta(\pi / 2) 10^{-2} 9^{-1}
$$

Let

$$
L \in\left(0, L_{0}\right], \rho>0, \sigma \in\left(0, \sigma_{0}\right] .
$$

Suppose that $S \geq L, \theta \in W^{1,2}(0, S)$ satisfies (3.1) and (1.7), $y:[0, S] \rightarrow R$ satisfies (1.6) and

$$
\int_{0}^{S}\left[\rho\left(\theta^{\prime}(t)\right)^{2}+\beta(\theta(t))+\sigma(y(t))^{2}\right] \mathrm{d} t=J_{\rho \sigma}^{L}(\theta, y)=\inf \left(J_{\rho \sigma}^{L}\right) .
$$

In order to prove Theorem 3.1 it is sufficient to show that there is no interval $[a, b] \subset[0, S]$ such that $a<b$ and $|\theta(t)|=\pi / 2$ for all $t \in[a, b]$.

Let us assume the converse. Then there is an interval $[a, b] \subset[0, S]$ such that $0<a<b<S$ and $|\theta(t)|=\pi / 2$ for all $t \in[a, b]$. We may assume without loss of generality that

$$
\theta(t)=\pi / 2 \text { for all } t \in[a, b]
$$

There is $\tau_{0} \in[0, S]$ such that

By (3.9) and (3.1)

$$
\theta\left(\tau_{0}\right)=\inf \{\theta(t): t \in[0, S]\} .
$$

$$
\theta\left(\tau_{0}\right) \leq-\pi / 2+r_{0}
$$

Corollary 2.1 implies that

By Lemma 2.3, (3.6) and (3.4)

$$
L \leq S \leq L \beta(0)(\beta(\pi / 2))^{-1} .
$$

$$
|y(t)| \leq \Delta_{0} \text { for all } t \in[0, S] .
$$

By continuity it follows from (3.10) and (3.3) that there is a positive number $\delta_{1}$ such that

$$
\begin{gathered}
\delta_{1}<(b-a) / 16 \\
\left.\left.\theta(t)<-\pi / 2+r_{1} \text { for all } t \in[0, S] \cap\right] \tau_{0}-2 \delta_{1}, \tau_{0}+2 \delta_{1}\right] .
\end{gathered}
$$


It follows from (3.13) that

$$
\delta_{1} \leq \operatorname{meas}\left([0, S] \cap\left[\tau_{0}-\delta_{1}, \tau_{0}+\delta_{1}\right]\right) \leq 2 \delta_{1} .
$$

There are three cases: (1) $\tau_{0} \leq \delta_{1}$; (2) $\tau_{0} \geq S-\delta_{1}>4 \delta_{1}$ (see (3.13)); (3) $\delta_{1}<\tau_{0}<S-\delta_{1}$.

In the case (1) set

$$
\begin{gathered}
\tilde{a}=0, \tilde{b}=\tau_{0}+\delta_{1}, S_{1}=S-\tilde{b}+\tilde{a} \\
\theta_{1}(t)=\theta(t+\tilde{b}-\tilde{a}), t \in\left[0, S_{1}\right] .
\end{gathered}
$$

In the case (2) put

$$
\begin{gathered}
\tilde{b}=S, \tilde{a}=\tau_{0}-\delta_{1}, S_{1}=S-\tilde{b}+\tilde{a}, \\
\theta_{1}(t)=\theta(t), t \in\left[0, S_{1}\right] .
\end{gathered}
$$

Consider the case (3). Since $\theta$ is continuous and $\tau_{0}$ satisfies $(3.9)$, there exists a closed interval $[\tilde{a}, \tilde{b}] \subset[0, S]$ such that

$$
\begin{aligned}
& \delta_{1} \leq \tilde{b}-\tilde{a} \leq 2 \delta_{1}, \tau_{0} \in[\tilde{a}, \tilde{b}] \subset\left[\tau_{0}-\delta_{1}, \tau_{0}+\delta_{1}\right], \\
& \theta(\tilde{a})=\theta(\tilde{b}) .
\end{aligned}
$$

We set

$$
\begin{gathered}
S_{1}=S-\tilde{b}+\tilde{a} \\
\theta_{1}(t)=\theta(t), t \in[0, \tilde{a}], \theta_{1}(t)=\theta(t+\tilde{b}-\tilde{a}), t \in\left(\tilde{a}, S_{1}\right] .
\end{gathered}
$$

It is not difficult to see that in the all three cases $\theta_{1} \in W^{1,2}\left(0, S_{1}\right)$,

$$
\begin{gathered}
\delta_{1} \leq \tilde{b}-\tilde{a} \leq 2 \delta_{1}, \\
\tau_{0} \in[\tilde{a}, \tilde{b}] .
\end{gathered}
$$

Relations (3.23), (3.24) and (3.14) imply that

$$
\theta(t) \leq-\pi / 2+r_{1} \text { for all } t \in[\tilde{a}, \tilde{b}]
$$

Clearly one of the following conditions holds:

$$
\tilde{a}=0 ; \tilde{b}=S ; \tilde{a}>0, \tilde{b}<S \text { and } \theta(\tilde{a})=\theta(\tilde{b}) .
$$

Define $y_{1}:\left[0, S_{1}\right] \rightarrow R$ by

$$
y_{1}(\tau)=y(0)+\int_{0}^{\tau} \sin \left(\theta_{1}(t)\right) \mathrm{d} t, t \in\left[0, S_{1}\right] .
$$

It follows from the definition of $y_{1}$ (see (3.27)), $\theta_{1}$ (see (3.17), (3.19), (3.22)), Lemmas 2.6 and 2.7, (3.12), (3.11), (3.23), (3.6) and (3.4) that

$$
\begin{aligned}
\left|\int_{0}^{S}(y(t))^{2} \mathrm{~d} t-\int_{0}^{S_{1}}\left(y_{1}(t)\right)^{2} \mathrm{~d} t\right| & \leq(\tilde{b}-\tilde{a}) \Delta_{0}^{2}+(\tilde{b}-\tilde{a})\left(2 \Delta_{0}+\tilde{b}-\tilde{a}\right) S \\
& \leq(\tilde{b}-\tilde{a})\left[\Delta_{0}^{2}+\left(2 \Delta_{0}+\tilde{b}-\tilde{a}\right) L_{0} \beta(0)(\beta(\pi / 2))^{-1}\right] \\
& \leq 2 \delta_{1}\left[\Delta_{0}^{2}+\left(2 \Delta_{0}+2 \delta_{1}\right) \Delta_{0} / 16\right] .
\end{aligned}
$$

Combined with (3.13), (3.11), (3.6) and (3.4) this inequality implies that

$$
\begin{aligned}
\left|\int_{0}^{S}(y(t))^{2} \mathrm{~d} t-\int_{0}^{S_{1}}\left(y_{1}(t)\right)^{2} \mathrm{~d} t\right| & \leq 2 \delta_{1}\left[\Delta_{0}^{2}+16^{-1} \Delta_{0}\left(2 \Delta_{0}+S\right)\right] \\
& \leq 2 \delta_{1}\left[\Delta_{0}^{2}+16^{-1} \Delta_{0}\left(2 \Delta_{0}+L_{0} \beta(0)(\beta(\pi / 2))^{-1}\right)\right] \\
& \leq 2 \delta_{1}\left[\Delta_{0}^{2}+16^{-1} \Delta_{0}\left(3 \Delta_{0}\right)\right] \leq 3 \delta_{1} \Delta_{0}^{2}
\end{aligned}
$$


Therefore we have shown that

$$
\left|\int_{0}^{S}(y(t))^{2} \mathrm{~d} t-\int_{0}^{S_{1}}\left(y_{1}(t)\right)^{2} \mathrm{~d} t\right| \leq 3 \delta_{1} \Delta_{0}^{2} .
$$

It follows from the definition of $\theta_{1}$ (see (3.17), (3.19), (3.22)), (3.25) and (3.8) that there are numbers $a_{1}, b_{1}$ such that

$$
\begin{gathered}
a_{1}, b_{1} \in\left[0, S_{1}\right], a_{1}<b_{1}<S_{1}, b_{1}-a_{1}=b-a, \\
\theta_{1}(t)=\pi / 2 \text { for all } t \in\left[a_{1}, b_{1}\right]
\end{gathered}
$$

Set

$$
\delta_{2}=-\int_{\tilde{a}}^{\tilde{b}} \sin (\theta(t)) \mathrm{d} t .
$$

Relations (3.31) and (3.23) imply that

$$
\delta_{2} \leq \tilde{b}-\tilde{a} \leq 2 \delta_{1}
$$

By (3.25) and (3.2) for each $t \in[\tilde{a}, \tilde{b}]$

$$
-\sin \theta(t) \geq \sin \left(\pi / 2-r_{1}\right) \geq \sin (\pi / 2-\pi / 16) \geq 1 / 2 .
$$

In view of (3.33), (3.31) and (3.23)

Together with (3.32) this implies that

$$
\delta_{2} \geq 2^{-1}(\tilde{b}-\tilde{a}) \geq \delta_{1} / 2 .
$$

$$
\delta_{1} / 2 \leq \delta_{2} \leq 2 \delta_{1}
$$

Set

$$
S_{2}=S_{1}-\delta_{2}
$$

Relations (3.35), (3.34), (3.16), (3.18), (3.21), (3.23) and (3.13) imply that

$$
S_{2} \geq S_{1}-2 \delta_{1}=S-\tilde{b}+\tilde{a}-2 \delta_{1} \geq S-4 \delta_{1} \geq S / 2 .
$$

Define $\theta_{2} \in W^{1,2}\left(0, S_{2}\right)$ by

$$
\theta_{2}(t)=\theta_{1}(t), t \in\left[0, b_{1}-\delta_{2}\right], \theta_{2}(t)=\theta_{1}\left(t+\delta_{2}\right), t \in\left(b_{1}-\delta_{2}, S_{2}\right]
$$

(see (3.30), (3.29), (3.13) and (3.34)).

Define $y_{2}:\left[0, S_{2}\right] \rightarrow R$ as follows:

$$
y_{2}(\tau)=y_{1}(0)+\int_{0}^{\tau} \sin \left(\theta_{2}(t)\right) \mathrm{d} t, \tau \in\left[0, S_{2}\right] .
$$

Relations (3.38) and (3.27) imply that

$$
y_{2}(0)=y_{1}(0)=y(0) .
$$

Combined with (3.12) this equality implies that

$$
\left|y_{2}(0)\right|=\left|y_{1}(0)\right|=|y(0)| \leq \Delta_{0}
$$

By $(3.40),(3.27),(3.11),(3.6)$ and $(3.4)$ for each $t \in\left[0, S_{1}\right]$

$$
\left|y_{1}(t)\right| \leq \Delta_{0}+t \leq \Delta_{0}+S \leq \Delta_{0}+L_{0} \beta(0)(\beta(\pi / 2))^{-1} \leq 2 \Delta_{0} .
$$

It follows from $(3.38),(3.40),(3.35),(3.11),(3.6)$ and $(3.4)$ that for each $t \in\left[0, S_{2}\right]$

$$
\left|y_{2}(t)\right| \leq \Delta_{0}+S \leq \Delta_{0}+L_{0} \beta(0)(\beta(\pi / 2))^{-1} \leq 2 \Delta_{0} .
$$


In view of (3.29), (3.13), (3.34) and (3.30)

$$
0 \leq a_{1}<b_{1}-\delta_{2}<b_{1}<S_{1}, \theta_{1}\left(b_{1}-\delta_{2}\right)=\theta_{1}\left(b_{1}\right)=\pi / 2
$$

It follows from the definition of $y_{2}$ (see (3.38)), $\theta_{2}$ (see (3.37)), (3.27), (3.41), (3.43), (3.35) and Lemma 2.7 (with $a=b_{1}-\delta_{2}, b=b_{1}, \theta=\theta_{1}$ ) that

$$
\left|\int_{0}^{S_{1}}\left(y_{1}(t)\right)^{2} \mathrm{~d} t-\int_{0}^{S_{2}}\left(y_{2}(t)\right)^{2} \mathrm{~d} t\right| \leq \delta_{2}\left(2 \Delta_{0}\right)^{2}+\delta_{2} S_{1}\left(4 \Delta_{0}+\delta_{2}\right) .
$$

Combined with (3.34), (3.13), (3.11), (3.6) and (3.4) this inequality implies that

$$
\begin{aligned}
\left|\int_{0}^{S_{1}}\left(y_{1}(t)\right)^{2} \mathrm{~d} t-\int_{0}^{S_{2}}(y(t))^{2} \mathrm{~d} t\right| & \leq 2 \delta_{1}\left[4 \Delta_{0}^{2}+S\left(4 \Delta_{0}+2 \delta_{1}\right)\right] \\
& \leq 2 \delta_{1}\left[4 \Delta_{0}^{2}+L_{0} \beta(0)(\beta(\pi / 2))^{-1}\left(4 \Delta_{0}+L_{0} \beta(0)(\beta(\pi / 2))^{-1}\right)\right] \\
& \leq 2 \delta_{1}\left[4 \Delta_{0}^{2}+(5 / 16) \Delta_{0}^{2}\right] \leq 10 \delta_{1} \Delta_{0}^{2} .
\end{aligned}
$$

Relations (3.44) and (3.28) imply that

$$
\left|\int_{0}^{S}(y(t))^{2} \mathrm{~d} t-\int_{0}^{S_{2}}\left(y_{2}(t)\right)^{2} \mathrm{~d} t\right| \leq 13 \delta_{1} \Delta_{0}^{2} .
$$

By (3.37), (3.35), (3.43), (3.30) and (3.31),

$$
\begin{gathered}
\int_{0}^{S_{2}} \sin \left(\theta_{2}(t)\right) \mathrm{d} t=\int_{0}^{b_{1}-\delta_{2}} \sin \left(\theta_{1}(t)\right) \mathrm{d} t+\int_{b_{1}}^{S_{1}} \sin \left(\theta_{1}(t)\right) \mathrm{d} t=\int_{0}^{S_{1}} \sin \left(\theta_{1}(t)\right) \mathrm{d} t \\
-\int_{b_{1}-\delta_{2}}^{b_{1}} \sin \left(\theta_{1}(t)\right) \mathrm{d} t=\int_{0}^{S_{1}} \sin \left(\theta_{1}(t)\right) \mathrm{d} t-\delta_{2}=\int_{0}^{S_{1}} \sin \left(\theta_{1}(t)\right) \mathrm{d} t+\int_{\tilde{a}}^{\tilde{b}} \sin (\theta(t)) \mathrm{d} t .
\end{gathered}
$$

It follows from this equality, the definition of $\theta_{1}$ (see (3.17), (3.19), (3.22)) and (1.7) that

$$
\int_{0}^{S_{2}} \sin \left(\theta_{2}(t)\right) \mathrm{d} t=\int_{0}^{S} \sin (\theta(t)) \mathrm{d} t=0 .
$$

By (3.29), (3.30), (3.43), (3.34), (3.13) and (3.37)

$$
\sup \left\{\theta_{2}(t): t \in\left[0, S_{2}\right]\right\}=\pi / 2 .
$$

Combined with (3.46) and the mean-value theorem this equality implies that there is $\tau_{*} \in\left[0, S_{2}\right]$ such that

$$
\theta_{2}\left(\tau_{*}\right)=0 .
$$

Set

By (3.49) and (3.23)

$$
\delta_{3}=\int_{\tilde{a}}^{\tilde{b}} \cos (\theta(t)) \mathrm{d} t
$$

$$
\delta_{3} \leq \tilde{b}-\tilde{a} \leq 2 \delta_{1}
$$


Set

and define $\theta_{3} \in W^{1,2}\left(0, S_{3}\right)$ by

$$
S_{3}=S_{2}+\delta_{3}
$$

$$
\begin{gathered}
\theta_{3}(t)=\theta_{2}(t), t \in\left[0, \tau_{*}\right], \theta_{3}(t)=0, t \in\left(\tau_{*}, \tau_{*}+\delta_{3}\right], \\
\theta_{3}(t)=\theta_{2}\left(t-\delta_{3}\right), t \in\left(\tau_{*}+\delta_{3}, S_{3}\right]
\end{gathered}
$$

(see (3.48)). Define

$$
y_{3}(\tau)=y_{2}(0)+\int_{0}^{\tau} \sin \left(\theta_{3}(t)\right) \mathrm{d} t, \tau \in\left[0, S_{3}\right] .
$$

In view of (3.52), (3.51) and (3.46)

$$
\int_{0}^{S_{3}} \sin \left(\theta_{3}(t)\right) \mathrm{d} t=\int_{0}^{\tau_{*}} \sin \left(\theta_{2}(t)\right) \mathrm{d} t+\int_{\tau_{*}}^{S_{2}} \sin \left(\theta_{2}(t)\right) \mathrm{d} t=0
$$

It follows from (3.52) and (3.51) that

$$
\int_{0}^{S_{3}} \cos \left(\theta_{3}(t)\right) \mathrm{d} t=\delta_{3}+\int_{0}^{S_{2}} \cos \left(\theta_{2}(t)\right) \mathrm{d} t
$$

By (3.37), (3.35), (3.43), (3.30) and the definition of $\theta_{1}$ (see (3.17), (3.19), (3.22))

$$
\begin{aligned}
& \int_{0}^{S_{2}} \cos \left(\theta_{2}(t)\right) \mathrm{d} t=\int_{0}^{b_{1}-\delta_{2}} \cos \left(\theta_{1}(t)\right) \mathrm{d} t+\int_{b_{1}}^{S_{1}} \cos \left(\theta_{1}(t)\right) \mathrm{d} t=\int_{0}^{S_{1}} \cos \left(\theta_{1}(t)\right) \mathrm{d} t \\
& -\int_{b_{1}-\delta_{2}}^{b_{1}} \cos \left(\theta_{1}(t)\right) \mathrm{d} t=\int_{0}^{S_{1}} \cos \left(\theta_{1}(t)\right) \mathrm{d} t=\int_{0}^{S} \cos (\theta(t)) \mathrm{d} t-\int_{\tilde{a}}^{\tilde{b}} \cos (\theta(t)) \mathrm{d} t .
\end{aligned}
$$

Combined with (3.55), (3.49) and (1.7) this equality implies that

$$
\int_{0}^{S_{3}} \cos \left(\theta_{3}(t)\right) \mathrm{d} t=L
$$

It follows from Lemma 2.8 (with $\left.\theta=\theta_{2}, \tilde{\theta}=\theta_{3}\right),(3.42),(3.50)-(3.53),(3.13),(3.11)$ and (3.6) that

$$
\begin{aligned}
&\left|\int_{0}^{S_{3}}\left(y_{3}(t)\right)^{2} \mathrm{~d} t-\int_{0}^{S_{2}}\left(y_{2}(t)\right)^{2} \mathrm{~d} t\right| \leq \delta_{3}\left(2 \Delta_{0}+\delta_{3}\right)^{2}+\delta_{3} S_{2}\left(4 \Delta_{0}+\delta_{3}\right) \\
& \leq 2 \delta_{1}\left[\left(2 \Delta_{0}+2 \delta_{1}\right)^{2}+S\left(4 \Delta_{0}+2 \delta_{1}\right)\right] \leq 2 \delta_{1}\left[\left(2 \Delta_{0}+S\right)^{2}+S\left(4 \Delta_{0}+S\right)\right] \\
& \leq 2 \delta_{1}\left[\left(2 \Delta_{0}+L_{0} \beta(0)(\beta(\pi / 2))^{-1}\right)^{2}+L_{0} \beta(0)(\beta(\pi / 2))^{-1}\left(4 \Delta_{0}+L_{0} \beta(0)\left(\beta(\pi / 2)^{-1}\right)\right]\right.
\end{aligned}
$$

Combined with (3.4) this inequality implies that

$$
\left|\int_{0}^{S_{3}}\left(y_{3}(t)\right)^{2} \mathrm{~d} t-\int_{0}^{S_{2}}\left(y_{2}(t)\right)^{2} \mathrm{~d} t\right| \leq 2 \delta_{1}\left[\left(2 \Delta_{0}+\Delta_{0} / 16\right)^{2}+16^{-1} 5 \Delta_{0}^{2}\right] \leq 2 \delta_{1}\left(9 \Delta_{0}^{2}\right) \leq 18 \delta_{1} \Delta_{0}^{2} .
$$

Together with (3.45) this implies that

$$
\left|\int_{0}^{S_{3}}\left(y_{3}(t)\right)^{2} \mathrm{~d} t-\int_{0}^{S}(y(t))^{2} \mathrm{~d} t\right| \leq 13 \delta_{1} \Delta_{0}^{2}+18 \delta_{1} \Delta_{0}^{2}=31 \delta_{1} \Delta_{0}^{2}
$$


We will estimate $J_{\rho \sigma}^{L}(\theta, y)-J_{\rho \sigma}^{L}\left(\theta_{3}, y_{3}\right)$. It follows from the definition of $\theta_{1}, \theta_{2}, \theta_{3}$ (see (3.17), (3.19), (3.22), (3.37) and (3.52)) that

$$
\int_{0}^{S_{3}}\left(\theta_{3}^{\prime}(t)\right)^{2} \mathrm{~d} t=\int_{0}^{S_{2}}\left(\theta_{2}^{\prime}(t)\right)^{2} \mathrm{~d} t \leq \int_{0}^{S_{1}}\left(\theta_{1}^{\prime}(t)\right)^{2} \mathrm{~d} t \leq \int_{0}^{S}\left(\theta^{\prime}(t)\right)^{2} \mathrm{~d} t
$$

In view of (3.52) and (3.51)

$$
\int_{0}^{S_{3}} \beta\left(\theta_{3}(t)\right) \mathrm{d} t=\int_{0}^{\tau_{*}} \beta\left(\theta_{2}(t)\right) \mathrm{d} t+\delta_{3} \beta(0)+\int_{\tau_{*}}^{S_{2}} \beta\left(\theta_{2}(t)\right) \mathrm{d} t=\delta_{3} \beta(0)+\int_{0}^{S_{2}} \beta\left(\theta_{2}(t)\right) \mathrm{d} t .
$$

By (3.37), (3.35), (3.43) and (3.30)

$$
\begin{aligned}
\int_{0}^{S_{2}} \beta\left(\theta_{2}(t)\right) \mathrm{d} t & =\int_{0}^{b_{1}-\delta_{2}} \beta\left(\theta_{1}(t)\right) \mathrm{d} t+\int_{b_{1}}^{S_{1}} \beta\left(\theta_{1}(t)\right) \mathrm{d} t \\
& =\int_{0}^{S_{1}} \beta\left(\theta_{1}(t)\right) \mathrm{d} t-\int_{b_{1}-\delta_{2}}^{b_{1}} \beta\left(\theta_{1}(t)\right) \mathrm{d} t=\int_{0}^{S_{1}} \beta\left(\theta_{1}(t)\right) \mathrm{d} t-\delta_{2} \beta(\pi / 2) .
\end{aligned}
$$

It follows from the definition of $\theta_{1}$ (see (3.17), (3.19) and (3.22)) that

$$
\int_{0}^{S_{1}} \beta\left(\theta_{1}(t)\right) \mathrm{d} t=\int_{0}^{S} \beta(\theta(t)) \mathrm{d} t-\int_{\tilde{a}}^{\tilde{b}} \beta(\theta(t)) \mathrm{d} t
$$

Equalitites (3.59)-(3.61) imply that

$$
\int_{0}^{S_{3}} \beta\left(\theta_{3}(t)\right) \mathrm{d} t=\delta_{3} \beta(0)-\delta_{2} \beta(\pi / 2)+\int_{0}^{S} \beta(\theta(t)) \mathrm{d} t-\int_{\tilde{a}}^{\tilde{b}} \beta(\theta(t)) \mathrm{d} t .
$$

Relations (3.49), (3.25), (3.22) and (3.2) imply that

$$
\delta_{3} \leq(\tilde{b}-\tilde{a}) \cos \left(\pi / 2-r_{1}\right) \leq 2 \delta_{1} \cos \left(\pi / 2-r_{1}\right) .
$$

By (3.62), (3.63), (3.34) and (3.2)

$$
\int_{0}^{S_{3}} \beta\left(\theta_{3}(t)\right) \mathrm{d} t-\int_{0}^{S} \beta(\theta(t)) \mathrm{d} t \leq \delta_{3} \beta(0)-\delta_{2} \beta(\pi / 2) \leq 2 \delta_{1} \cos \left(\pi / 2-r_{1}\right) \beta(0)-2^{-1} \delta_{1} \beta(\pi / 2) \leq-4^{-1} \delta_{1} \beta(\pi / 2) .
$$

In view of (1.5), (3.64), (3.58), (3.57), (3.6) and (3.5)

$$
\begin{aligned}
& J_{\rho \sigma}^{L}\left(\theta_{3}, y_{3}\right)-J_{\rho \sigma}^{L}(\theta, y)=\int_{0}^{S_{3}} \beta\left(\theta_{3}(t)\right) \mathrm{d} t-\int_{0}^{S} \beta(\theta(t)) \mathrm{d} t \\
&+\rho\left[\int_{0}^{S_{3}}\left(\theta_{3}^{\prime}(t)\right)^{2} \mathrm{~d} t-\int_{0}^{S}\left(\theta^{\prime}(t)\right)^{2} \mathrm{~d} t\right]+\sigma\left[\int_{0}^{S_{3}}\left(y_{3}(t)\right)^{2} \mathrm{~d} t-\int_{0}^{S}(y(t))^{2} \mathrm{~d} t\right] \\
& \quad \leq-4^{-1} \delta_{1} \beta(\pi / 2)+31 \sigma \delta_{1} \Delta_{0}^{2} \leq-4^{-1} \delta_{1} \beta(\pi / 2)+31 \sigma_{0} \delta_{1} \Delta_{0}^{2}<0,
\end{aligned}
$$

a contradiction. The contradiction we have reached proves Theorem 3.1. 


\section{Proof of Theorem 1.1}

By Theorem 3.1 there are

$$
r_{0} \in(0, \pi / 8), \sigma_{0}>0
$$

such that the following assertion holds:

(A1) If $L \in\left(0, L_{1}\right], \rho>0, \sigma \in\left(0, \sigma_{0}\right]$ and if $(S, \theta, y)$ is a solution of the problem (1.5)-(1.7) satisfying

$$
\left[-\pi / 2+r_{0}, \pi / 2-r_{0}\right] \subset \theta([0, S])
$$

then there is no interval $[a, b] \subset[0, S]$ such that $a<b$ and $|\theta(t)|=\pi / 2$ for all $t \in[a, b]$.

Choose a positive number $\gamma$ such that

$$
\gamma \leq \arcsin \left(2^{-1}(\beta(0))^{-1} \beta(\pi / 2) \min \left\{1,16^{-1} \pi^{2} \rho_{1} L_{1}^{-2}(\beta(0))^{-1}\right\}\right)
$$

and choose a number $\sigma_{1}$ such that

$$
\begin{gathered}
0<\sigma_{1}<\sigma_{0}, \\
\sigma_{1}\left(1+\left(\cos \left(\pi / 2-r_{0}\right)\right)^{-1}\right)\left(16 L_{1} \beta(0)(\beta(\pi / 2))^{-1}+4\right)^{3}<\sin (\gamma) \beta(\pi / 2) / 3200
\end{gathered}
$$

Let

$$
L \in\left(0, L_{1}\right], \rho \geq \rho_{1}, \sigma \in\left(0, \sigma_{1}\right]
$$

Suppose that $S \geq L, \theta \in W^{1,2}(0, S)$ satisfies (1.7) and $y:[0, S] \rightarrow R$ satisfies (1.6) and

$$
\int_{0}^{S}\left[\rho\left(\theta^{\prime}(t)\right)^{2}+\beta(\theta(t))+\sigma(y(t))^{2}\right] \mathrm{d} t=J_{\rho \sigma}^{L}(\theta, y)=\inf \left(J_{\rho \sigma}^{L}\right) .
$$

In order to prove Theorem 1.1 it is sufficient to show that there is no interval $[a, b] \subset[0, S]$ such that $a<b$ and $|\theta(t)|=\pi / 2$ for all $t \in[a, b]$.

Let us assume the converse. Then there is an interval $[a, b] \subset[0, S]$ such that $0<a<b<S$ and $|\theta(t)|=\pi / 2$ for all $t \in[a, b]$.

We may assume without loss of generality that

$$
\theta(t)=\pi / 2 \text { for all } t \in[a, b] .
$$

It follows from Lemma 2.5, (4.3), (4.6) and (4.8) that

$$
\min \{\theta(t): t \in[0, S]\} \leq-\gamma
$$

Corollary 2.1 implies that

$$
L \leq S \leq L \beta(0)(\beta(\pi / 2))^{-1} .
$$

In view of (4.8), assertion (A1), (4.4) and (4.6) the inclusion (4.2) does not hold. Together with (4.8) this implies that

$$
\min \{\theta(t): t \in[0, S]\} \geq-\pi / 2+r_{0}
$$

Choose a positive number $\epsilon$ such that

$$
\epsilon<\gamma / 4 \text { and } \epsilon\left(\beta(0) \cos \left(\pi / 2-r_{0}\right)^{-1}+1\right)<\beta(\pi / 2) \sin (\gamma) / 4
$$

There is a positive number $\delta$ such that

$$
\begin{gathered}
\delta<\min \left\{1 / 8, L / 16,32^{-1}(b-a) \cos \left(\pi / 2-r_{0}\right)\right\} \\
\delta\left(1+\cos \left(\pi / 2-r_{0}\right)^{-1}\right)<1 / 8
\end{gathered}
$$


and

$$
\begin{aligned}
& \text { if } t_{1}, t_{2} \in[0, S] \text { satisfies }\left|t_{1}-t_{2}\right| \leq 2 \delta, \text { then } \\
& \left|\theta\left(t_{1}\right)-\theta\left(t_{2}\right)\right| \leq \epsilon,\left|\beta\left(\theta\left(t_{1}\right)\right)-\beta\left(\theta\left(t_{2}\right)\right)\right| \leq \epsilon
\end{aligned}
$$

There is $t_{0} \in[0, S]$ such that

$$
\theta\left(t_{0}\right)=\inf \{\theta(t): t \in[0, S]\} .
$$

There are three cases: (1) $t_{0} \leq \delta$; (2) $t_{0} \geq S-\delta$; (3) $\delta<t_{0}<S-\delta$. In the case (1) set

$$
\begin{gathered}
c=0, d=t_{0}+\delta, S_{1}=S-d, \\
\theta_{1}(t)=\theta(t+d), t \in\left[0, S_{1}\right] .
\end{gathered}
$$

In the case (2) put

$$
\begin{gathered}
d=S, c=t_{0}-\delta, S_{1}=t_{0}-\delta, \\
\theta_{1}(t)=\theta(t), t \in\left[0, S_{1}\right] .
\end{gathered}
$$

Consider the case (3). Since $\theta$ is continuous and $t_{0}$ satisfies (4.16), there exists a closed interval $[c, d] \subset[0, S]$ such that

$$
\begin{gathered}
\delta \leq d-c \leq 2 \delta, \\
t_{0} \in[c, d] \subset\left[t_{0}-\delta, t_{0}+\delta\right], \\
\theta(c)=\theta(d) .
\end{gathered}
$$

We set

$$
\begin{gathered}
S_{1}=S-d+c \\
\theta_{1}(t)=\theta(t), t \in[0, c], \theta_{1}(t)=\theta(t+d-c], t \in\left(c, S_{1}\right] .
\end{gathered}
$$

It is not difficult to see that in all three cases $\theta_{1} \in W^{1,2}\left(0, S_{1}\right),(4.21),(4.22)$ are true and one of the following conditions holds:

$$
c=0 ; d=S ; c>0, d<S \text { and } \theta(c)=\theta(d) .
$$

In view of (4.22) and the choice of $\delta$ (see (4.15)) for each $t \in[c, d]$

$$
\left|\theta(t)-\theta\left(t_{0}\right)\right| \leq \epsilon,\left|\beta(\theta(t))-\beta\left(\theta\left(t_{0}\right)\right)\right| \leq \epsilon .
$$

By (4.16), (4.27), (4.9) and (4.12) for each $t \in[c, d]$

$$
\theta\left(t_{0}\right) \leq \theta(t) \leq \theta\left(t_{0}\right)+\epsilon \leq-\gamma+\epsilon \leq-(3 / 4) \gamma .
$$

Inequality (4.28) implies that

$$
\int_{c}^{d} \cos (\theta(t)) \mathrm{d} t \geq(d-c) \cos \left(\theta\left(t_{0}\right)\right)
$$

In view of $(4.16),(4.11)$ and $(4.9) \cos \left(\theta\left(t_{0}\right)\right) \neq 0$. Set

$$
\Delta_{0}=\left(\int_{c}^{d} \cos (\theta(t)) \mathrm{d} t\right)\left(\cos \left(\theta\left(t_{0}\right)\right)\right)^{-1} .
$$

By (4.30), (4.29), (4.9), (4.11) and (4.16)

$$
d-c \leq \Delta_{0} \leq(d-c)\left(\cos \left(\theta\left(t_{0}\right)\right)\right)^{-1} \leq(d-c)\left(\cos \left(\pi / 2-r_{0}\right)\right)^{-1} .
$$

It follows from (4.8), (4.28), and the construction of $\theta_{1}$ (see (4.18), (4.20), (4.25)) that there is an interval $\left[a_{1}, b_{1}\right] \subset\left[0, S_{1}\right]$ such that

$$
b_{1}<S_{1}, b_{1}-a_{1}=b-a, \theta_{1}(t)=\pi / 2, t \in\left[a_{1}, b_{1}\right] .
$$


In view of (4.28) and the definition of $\theta_{1}$ (see (4.18), (4.20), (4.25))

$$
\inf \left\{\theta_{1}(t): t \in\left[0, S_{1}\right]\right\} \leq-(3 / 4) \gamma .
$$

By (4.32), (4.33) and (4.28) there is $t_{1} \in\left[0, S_{1}\right]$ such that

$$
\theta_{1}\left(t_{1}\right)=-\theta\left(t_{0}\right)
$$

Set

and define

$$
S_{2}=S_{1}+\Delta_{0}
$$

$$
\begin{gathered}
\theta_{2}(t)=\theta_{1}(t), t \in\left[0, t_{1}\right], \theta_{2}(t)=\theta_{1}\left(t_{1}\right), t \in\left(t_{1}, t_{1}+\Delta_{0}\right], \\
\theta_{2}(t)=\theta_{1}\left(t-\Delta_{0}\right), t \in\left(t_{1}+\Delta_{0}, S_{2}\right] .
\end{gathered}
$$

Clearly $\theta_{2} \in W^{1,2}\left(0, S_{2}\right)$.

It follows from (4.36), (4.35), (4.34), (1,7), (4.30) and the definition of $\theta_{1}$ (see (4.18), (4.20), (4.25)) that

$$
\begin{aligned}
\int_{0}^{S_{2}} \cos \left(\theta_{2}(t)\right) \mathrm{d} t & =\int_{0}^{S_{1}} \cos \left(\theta_{1}(t)\right) \mathrm{d} t+\Delta_{0} \cos \left(\theta\left(t_{0}\right)\right) \\
& =\int_{0}^{S_{1}} \cos \left(\theta_{1}(t)\right) \mathrm{d} t+\int_{c}^{d} \cos (\theta(t)) \mathrm{d} t=\int_{0}^{S} \cos (\theta(t)) \mathrm{d} t=L .
\end{aligned}
$$

By (4.36), (4.34), (4.35), the definition of $\theta_{1}$ (see (4.18), (4.20), (4.25)) and (1.7)

$$
\int_{0}^{S_{2}} \sin \left(\theta_{2}(t)\right) \mathrm{d} t=\int_{0}^{S_{1}} \sin \left(\theta_{1}(t)\right) \mathrm{d} t-\Delta_{0} \sin \left(\theta\left(t_{0}\right)\right)=-\int_{c}^{d} \sin (\theta(t)) \mathrm{d} t-\Delta_{0} \sin \left(\theta\left(t_{0}\right)\right) .
$$

Set

$$
\Delta_{1}=-\int_{c}^{d} \sin (\theta(t)) \mathrm{d} t-\Delta_{0} \sin \left(\theta\left(t_{0}\right)\right)
$$

In view of (4.39), (4.28) and (4.30)

$$
0<\Delta_{1} \leq d-c+\Delta_{0}
$$

It follows from (4.32) and the construction of $\theta_{2}$ (see (4.35), (4.36)) that there is an interval $\left[a_{2}, b_{2}\right] \subset\left[0, S_{2}\right]$ such that

$$
b_{2}<S_{2}, b_{2}-a_{2}=b_{1}-a_{1}=b-a, \theta_{2}(t)=\pi / 2, t \in\left[a_{2}, b_{2}\right] .
$$

Relations (4.39), (4.31), (4.21) and (4.13) imply that

$$
\Delta_{1} \leq d-c-\Delta_{0} \sin \left(\theta\left(t_{0}\right)\right) \leq d-c+\Delta_{0} \leq 2(d-c)\left(\cos \left(\pi / 2-r_{0}\right)\right)^{-1} \leq 4 \delta\left(\cos \left(\pi / 2-r_{0}\right)\right)^{-1} \leq 8^{-1}(b-a) .
$$

Set

$$
S_{3}=S_{2}-\Delta_{1}
$$

Combined with (4.43), (4.35), (4.24), (4.21) and (4.13) the inequality (4.42) implies that

$$
S_{3} \geq S_{1}-\Delta_{1}=S-\Delta_{1}-d+c \geq S-\Delta_{1}-2 \delta \geq b-a-8^{-1}(b-a)-16^{-1}(b-a) \geq(3 / 4)(b-a) .
$$

Define

$$
\theta_{3}(t)=\theta_{2}(t), t \in\left[0, b_{2}-\Delta_{1}\right], \theta_{3}(t)=\theta_{2}\left(t+\Delta_{1}\right), t \in\left[b_{2}-\Delta_{1}, S_{3}\right]
$$


(see (4.41)). Clearly $\theta_{3} \in W^{1,2}\left(0, S_{3}\right)$. In view of (4.45), (4.43), (4.41), (4.42) and (4.37)

$$
\begin{aligned}
\int_{0}^{S_{3}} \cos \left(\theta_{3}(t)\right) \mathrm{d} t & =\int_{0}^{b_{2}-\Delta_{1}} \cos \left(\theta_{2}(t)\right) \mathrm{d} t+\int_{b_{2}}^{S_{2}} \cos \left(\theta_{2}(t)\right) \mathrm{d} t \\
& =\int_{0}^{S_{2}} \cos \left(\theta_{2}(t)\right) \mathrm{d} t-\int_{b_{2}-\Delta_{1}}^{b_{2}} \cos \left(\theta_{2}(t)\right) \mathrm{d} t=\int_{0}^{S_{2}} \cos \left(\theta_{2}(t)\right) \mathrm{d} t=L .
\end{aligned}
$$

By (4.45), (4.43), (4.41), (4.42), (4.38) and (4.39)

$$
\begin{aligned}
\int_{0}^{S_{3}} \sin \left(\theta_{3}(t)\right) \mathrm{d} t & =\int_{0}^{b_{2}-\Delta_{1}} \sin \left(\theta_{2}(t)\right) \mathrm{d} t+\int_{b_{2}}^{S_{2}} \sin \left(\theta_{2}(t)\right) \mathrm{d} t \\
& =\int_{0}^{S_{2}} \sin \left(\theta_{2}(t)\right) \mathrm{d} t-\Delta_{1}=0 .
\end{aligned}
$$

It follows from the definition of $\theta_{1}, \theta_{2}, \theta_{3}$ (see (4.18), (4.20), (4.25), (4.36), (4.45)) that

$$
\int_{0}^{S_{3}}\left(\theta_{3}^{\prime}(t)\right)^{2} \mathrm{~d} t \leq \int_{0}^{S_{2}}\left(\theta_{2}^{\prime}(t)\right)^{2} \mathrm{~d} t=\int_{0}^{S_{1}}\left(\theta_{1}^{\prime}(t)\right)^{2} \mathrm{~d} t \leq \int_{0}^{S}\left(\theta^{\prime}(t)\right)^{2} \mathrm{~d} t .
$$

We estimate

$$
\int_{0}^{S_{3}} \beta\left(\theta_{3}(t)\right) \mathrm{d} t-\int_{0}^{S} \beta(\theta(t)) \mathrm{d} t
$$

By $(4.45)$ and (4.43)

$$
\begin{aligned}
\int_{0}^{S_{3}} \beta\left(\theta_{3}(t)\right) \mathrm{d} t & =\int_{0}^{b_{2}-\Delta_{1}} \beta\left(\theta_{2}(t)\right) \mathrm{d} t+\int_{b_{2}}^{S_{2}} \beta\left(\theta_{2}(t)\right) \mathrm{d} t \\
& =\int_{0}^{S_{2}} \beta\left(\theta_{2}(t)\right) \mathrm{d} t-\int_{b_{2}-\Delta_{1}}^{b_{2}} \beta\left(\theta_{2}(t)\right) \mathrm{d} t .
\end{aligned}
$$

Combined with (4.41), (4.42), (4.36), (4.34), (4.35) and (1.2) this equality implies that

$$
\begin{aligned}
\int_{0}^{S_{3}} \beta\left(\theta_{3}(t)\right) \mathrm{d} t & =\int_{0}^{S_{2}} \beta\left(\theta_{2}(t)\right) \mathrm{d} t-\Delta_{1} \beta(\pi / 2) \\
& =-\Delta_{1} \beta(\pi / 2)+\Delta_{0} \beta\left(\theta\left(t_{0}\right)\right)+\int_{0}^{t_{1}} \beta\left(\theta_{1}(t)\right) \mathrm{d} t+\int_{t_{1}}^{S_{1}} \beta\left(\theta_{1}(t)\right) \mathrm{d} t \\
& =\Delta_{1} \beta(\pi / 2)+\Delta_{0} \beta\left(\theta\left(t_{0}\right)\right)+\int_{0}^{S_{1}} \beta\left(\theta_{1}(t)\right) \mathrm{d} t .
\end{aligned}
$$

Together with the definition of $\theta_{1}$ (see (4.18), (4.20), (4.25)) this equality implies that

$$
\int_{0}^{S_{3}} \beta\left(\theta_{3}(t)\right) \mathrm{d} t=-\Delta_{1} \beta(\pi / 2)+\Delta_{0} \beta\left(\theta\left(t_{0}\right)\right)+\int_{0}^{S} \beta(\theta(t)) \mathrm{d} t-\int_{c}^{d} \beta(\theta(t)) \mathrm{d} t .
$$

Thus

$$
\int_{0}^{S_{3}} \beta\left(\theta_{3}(t)\right) \mathrm{d} t-\int_{0}^{S} \beta(\theta(t)) \mathrm{d} t=-\Delta_{1} \beta(\pi / 2)+\Delta_{0} \beta\left(\theta\left(t_{0}\right)\right)-\int_{c}^{d} \beta(\theta(t)) \mathrm{d} t .
$$


In view of (1.3) and (4.27)

$$
\begin{aligned}
\Delta_{0} \beta\left(\theta\left(t_{0}\right)\right)-\int_{c}^{d} \beta(\theta(t)) \mathrm{d} t=\beta\left(\theta\left(t_{0}\right)\right)\left[\Delta_{0}-(d-c)\right] \\
\\
+\int_{c}^{d}\left[\beta\left(\theta\left(t_{0}\right)\right)-\beta(\theta(t))\right] \mathrm{d} t \leq \beta(0)\left[\Delta_{0}-d+c\right]+\epsilon(d-c) .
\end{aligned}
$$

By (4.30), (4.27), (4.16), (4.9) and (4.11)

$$
\begin{aligned}
\Delta_{0}-d+c & =\left(\cos \left(\theta\left(t_{0}\right)\right)\right)^{-1} \int_{c}^{d}\left[\cos (\theta(t))-\cos \left(\theta\left(t_{0}\right)\right)\right] \mathrm{d} t \\
& \leq\left(\cos \left(\theta\left(t_{0}\right)\right)\right)^{-1} \int_{c}^{d}\left|\theta(t)-\theta\left(t_{0}\right)\right| \mathrm{d} t \\
& \left.\leq\left(\cos \left(\theta\left(t_{0}\right)\right)\right)^{-1} \epsilon(d-c) \leq \epsilon(d-c)\left(\cos \left(\pi / 2-r_{0}\right)\right)\right)^{-1}
\end{aligned}
$$

Combined with (4.50) the relation (4.51) implies that

$$
\Delta_{0} \beta\left(\theta\left(t_{0}\right)\right)-\int_{c}^{d} \beta(\theta(t)) \mathrm{d} t \leq \beta(0)(d-c)\left(\cos \left(\pi / 2-r_{0}\right)\right)^{-1} \epsilon+\epsilon(d-c)=(d-c)\left[\beta(0) \epsilon\left(\cos \left(\pi / 2-r_{0}\right)\right)^{-1}+\epsilon\right] .
$$

Relations (4.39), (4.28), (4.31), (4.16) and (4.9) imply that

$$
\Delta_{1} \geq-\Delta_{0} \sin \left(\theta\left(t_{0}\right)\right) \geq-\sin \left(\theta\left(t_{0}\right)\right)(d-c) \geq(d-c) \sin (\gamma)
$$

Together with (4.49), (4.52) and (4.12) this inequality implies that

$$
\begin{array}{r}
\int_{0}^{S_{3}} \beta\left(\theta_{3}(t)\right) \mathrm{d} t-\int_{0}^{S} \beta(\theta(t)) \mathrm{d} t \\
\leq(d-c)\left[-\beta(\pi / 2) \sin (\gamma)+\epsilon\left(\beta(0)\left(\cos \left(\pi / 2-r_{0}\right)\right)^{-1}+1\right)\right] \\
\leq-(d-c) \sin (\gamma) \beta(\pi / 2) / 2
\end{array}
$$

For $i=1,2,3$ set

$$
y_{i}(\tau)=y(0)+\int_{0}^{\tau} \sin \left(\theta_{i}(t)\right) \mathrm{d} t, \tau \in\left[0, S_{i}\right]
$$

We estimate $\int_{0}^{S}(y(t))^{2} \mathrm{~d} t-\int_{0}^{S_{3}}\left(y_{3}(t)\right)^{2} \mathrm{~d} t$. Lemma 2.3 implies that

$$
|y(t)| \leq 8 L \beta(0)(\beta(\pi / 2))^{-1}, t \in[0, S]
$$

It follows from (4.55), (4.54), the definition of $\theta_{1}$ (see (4.18), (4.20), (4.25)), Lemmas 2.6 and 2.7 and (4.10) that

$$
\begin{aligned}
\left|\int_{0}^{S_{1}}\left(y_{1}(t)\right)^{2} \mathrm{~d} t-\int_{0}^{S}(y(t))^{2} \mathrm{~d} t\right| \leq & (d-c)\left(8 L \beta(0)(\beta(\pi / 2))^{-1}\right)^{2} \\
& +(d-c) S\left(16 L \beta(0)(\beta(\pi / 2))^{-1}+d-c\right) \\
\leq & (d-c)\left[64\left(L \beta(0)(\beta(\pi / 2))^{-1}\right)^{2}+17\left(L \beta(0)(\beta(\pi / 2))^{-1}\right)^{2}\right] \\
= & (d-c) 81\left(L \beta(0)(\beta(\pi / 2))^{-1}\right)^{2}
\end{aligned}
$$


Relations (4.54), (4.55), (4.24), (4.10) and (4.13) imply that for all $t \in\left[0, S_{1}\right]$

$$
\left|y_{1}(t)\right| \leq|y(0)|+t \leq|y(0)|+S_{1} \leq 8 L \beta(0)(\beta(\pi / 2))^{-1}+S \leq 9 L \beta(0)(\beta(\pi / 2))^{-1} .
$$

Combined with (4.54), (4.36), Lemma 2.8, (4.31), (4.22), (4.10) and (4.13) this inequality implies that

$$
\begin{aligned}
\left|\int_{0}^{S_{2}}\left(y_{2}(t)\right)^{2} \mathrm{~d} t-\int_{0}^{S_{1}}\left(y_{1}(t)\right)^{2} \mathrm{~d} t\right| \leq & \Delta_{0}\left(9 L \beta(0)(\beta(\pi / 2))^{-1}+\Delta_{0}\right)^{2} \\
& +\Delta_{0} S_{1}\left(\Delta_{0}+18 L \beta(0)(\beta(\pi / 2))^{-1}\right) \\
\leq & \Delta_{0}\left[\left(10 L \beta(0)(\beta(\pi / 2))^{-1}\right)^{2}+19\left(L \beta(0)(\beta(\pi / 2))^{-1}\right)^{2}\right] \\
\leq & 29(d-c)\left(\cos \left(\pi / 2-r_{0}\right)\right)^{-1}\left(L \beta(0)(\beta(\pi / 2))^{-1}\right)^{2} .
\end{aligned}
$$

In view of (4.35), (4.31), (4.21) and (4.13)

$$
\begin{aligned}
S_{2} & =S_{1}+\Delta_{0} \leq S+(d-c)\left(\cos \left(\pi / 2-r_{0}\right)\right)^{-1} \\
& \leq S+2 \delta\left(\cos \left(\pi / 2-r_{0}\right)\right)^{-1} \leq S+b-a \leq 2 S .
\end{aligned}
$$

By (4.54), (4.55), (4.58) and (4.10) for each $t \in\left[0, S_{2}\right]$

$$
\left|y_{2}(t)\right| \leq|y(0)|+t \leq|y(0)|+S_{2} \leq 8 L \beta(0)(\beta(\pi / 2))^{-1}+2 S \leq 10 L \beta(0)(\beta(\pi / 2))^{-1} .
$$

It follows from $(4.54),(4.58),(4.59),(4.45),(4.40)-(4.42)$, Lemma 2.7 and (4.10) that

$$
\begin{aligned}
\left|\int_{0}^{S_{2}}\left(y_{2}(t)\right)^{2} \mathrm{~d} t-\int_{0}^{S_{3}}\left(y_{3}(t)\right)^{2} \mathrm{~d} t\right| \leq & \Delta_{1}\left(10 L \beta(0)(\beta(\pi / 2))^{-1}\right)^{2} \\
& +\Delta_{1} S_{2}\left(20 L \beta(0)(\beta(\pi / 2))^{-1}+\Delta_{1}\right) \\
\leq & \Delta_{1}\left[10^{2}\left(L \beta(0)(\beta(\pi / 2))^{-1}\right)^{2}+2 S\left(20 L \beta(0)(\beta(\pi / 2))^{-1}+S\right)\right] \\
\leq & 142 \Delta_{1}\left[L \beta(0)(\beta(\pi / 2))^{-1}\right)^{2} .
\end{aligned}
$$

Together with (4.42) this implies that

$$
\left|\int_{0}^{S_{2}}\left(y_{2}(t)\right)^{2} \mathrm{~d} t-\int_{0}^{S_{3}}\left(y_{3}(t)\right)^{2} \mathrm{~d} t\right| \leq 142\left(L \beta(0)(\beta(\pi / 2))^{-1}\right)^{2} 2(d-c)\left(\cos \left(\pi / 2-r_{0}\right)\right)^{-1} .
$$

Combined with (4.57) and (4.56) this inequality implies that

$$
\begin{aligned}
\left|\int_{0}^{S}(y(t))^{2} \mathrm{~d} t-\int_{0}^{S_{3}}\left(y_{3}(t)\right)^{2} \mathrm{~d} t\right| \leq & 81(d-c)\left(L \beta(0)(\beta(\pi / 2))^{-1}\right)^{2} \\
& +29(d-c)\left(\cos \left(\pi / 2-r_{0}\right)\right)^{-1}\left(L \beta(0)(\beta(\pi / 2))^{-1}\right)^{2} \\
& +284(d-c)\left(\cos \left(\pi / 2-r_{0}\right)\right)^{-1}\left(L \beta(0)(\beta(\pi / 2))^{-1}\right)^{2} \\
\leq & 400(d-c)\left(L \beta(0)(\beta(\pi / 2))^{-1}\right)\left(\cos \left(\pi / 2-r_{0}\right)\right)^{-1}
\end{aligned}
$$


By (1.5), (4.60), (4.6), (4.48), (4.53) and (4.5)

$$
\begin{aligned}
J_{\rho \sigma}^{L}\left(\theta_{3}, y_{3}\right)-J_{\rho \sigma}^{L}(\theta, y)= & \sigma\left[\int_{0}^{S_{3}}\left(y_{3}(t)\right)^{2} \mathrm{~d} t-\int_{0}^{S}(y(t))^{2} \mathrm{~d} t\right] \\
& +\rho\left[\int_{0}^{S_{3}}\left(\theta_{3}^{\prime}(t)\right)^{2} \mathrm{~d} t-\int_{0}^{S}\left(\theta^{\prime}(t)\right)^{2} \mathrm{~d} t\right]+\int_{0}^{S_{3}} \beta\left(\theta_{3}(t)\right) \mathrm{d} t-\int_{0}^{S} \beta(\theta(t)) \mathrm{d} t \\
\leq & 400 \sigma_{1}\left((d-c)\left(L \beta(0)(\beta(\pi / 2))^{-1}\right)^{2}\left(\cos \left(\pi / 2-r_{0}\right)\right)^{-1}+\int_{0}^{S_{3}} \beta\left(\theta_{3}(t)\right) \mathrm{d} t-\int_{0}^{S} \beta(\theta(t)) \mathrm{d} t\right. \\
\leq & 400(d-c) \sigma_{1}\left(L_{1} \beta(0)(\beta(\pi / 2))^{-1}\right)^{2}\left(\cos \left(\pi / 2-r_{0}\right)\right)^{-1}-(d-c) \sin (\gamma) \beta(\pi / 2) / 2<0,
\end{aligned}
$$

a contradiction. The contradiction we have reached proves Theorem 1.1.

\section{The PARAmeter $\sigma_{1}$ AS A Function of $\beta, L_{1}$ AND $\rho_{1}$}

Let $\rho_{1}, L_{1}>0$. We proved the existence of a positive number $\sigma_{1}$ which depends on $\beta, L_{1}, \rho_{1}$ such that the assertion of Theorem 1.1 holds. In this section we obtain an explicit expression for $\sigma_{1}$ which is a function of $\beta$, $L_{1}, \rho_{1}$. We assume that

$$
L_{1}>1 \text { and } 16^{-1} \pi^{2} \rho_{1} L_{1}^{-2}(\beta(0))^{-1}<1
$$

set

$$
r_{1}=\beta(\pi / 2)(\beta(0))^{-1} 16^{-1}
$$

and observe that $r_{1}$ satisfies (3.2). Clearly

$$
0<r_{1}<\pi / 16
$$

so that

$$
r_{1} / 2 \leq \cos \left(\pi / 2-r_{1}\right) \leq r_{1}
$$

Relations (5.4) and (5.2) imply that

$$
\beta(0) \cos \left(\pi / 2-r_{1}\right)=r_{1} \beta(0) \leq \beta(\pi / 2) 16^{-1} .
$$

Together with (5.3) this implies that (3.2) holds. Put

$$
r_{0}=r_{1} / 3
$$

Then (3.3) is valid. Set

$$
\sigma_{0}=10^{-3} \beta(\pi / 2)\left[16 L_{1} \beta(0)(\beta(\pi / 2))^{-1}\right]^{-2} .
$$

Clearly (3.5) is true with $L_{0}=L_{1}$ and $\Delta_{0}=16 r_{1} \beta(0) \beta(\pi / 2)^{-1}$. We showed that the assertion of Theorem 3.1 holds with $L_{0}=L_{1}$. Thus (A1) holds (see Sect. 4). Now we need to choose positive numbers $\gamma, \sigma_{1}$. Set

$$
\gamma=\arcsin \left(2^{-1}(\beta(0))^{-1} \beta(\pi / 2)\right) \min \left\{1,16^{-1} \pi^{2} \rho_{1} L_{1}^{-2}(\beta(0))^{-1}\right\}
$$

Clearly (4.3) holds.

Finally we need to choose $\sigma_{1}>0$ which satisfies (4.4) and (4.5). Set

$$
\sigma_{1}=(\beta(0))^{-6} \beta(\pi / 2)^{6} L_{1}^{-5} \rho_{1}\left(17 \cdot 400 \cdot 16^{2} \cdot 20^{3}\right)^{-1} .
$$


It follows from (5.7), (5.5), (5.3), (5.2), (5.1) and (5.8) that

$$
\begin{gathered}
8^{-1} \sin (\gamma) \beta(\pi / 2)\left[1+\left(\cos \left(\pi / 2-r_{0}\right)\right)^{-1}\right]^{-1}\left(4+16 L_{1} \beta(0)(\beta(\pi / 2))^{-1}\right)^{-3} \\
=2^{-1}(\beta(0))^{-1} \beta(\pi / 2) \min \left\{1,16^{-1} \pi^{2} \rho_{1} L_{1}^{-2}(\beta(0))^{-1}\right\} 8^{-1} \beta(\pi / 2)[1 \\
\left.+\left(\cos \left(\pi / 2-r_{0}\right)\right)^{-1}\right]^{-1}\left(4+16 L_{1} \beta(0)(\beta(\pi / 2))^{-1}\right)^{-3} \\
\geq 2^{-1}(\beta(0))^{-1} \beta(\pi / 2) \min \left\{1, \pi^{2} 16^{-1} \rho_{1} L_{1}^{-2}(\beta(0))^{-1}\right\} \\
\times 8^{-1} \beta(\pi / 2)\left[1+2 r_{0}^{-1}\right]^{-1}\left(4+16 L_{1} \beta(0)(\beta(\pi / 2))^{-1}\right)^{-3} \\
\geq 2^{-1}(\beta(0))^{-1} \beta(\pi / 2) \min \left\{1,16^{-1} \pi^{2} \rho_{1} L_{1}^{-2}(\beta(0))^{-1}\right\} 8^{-1} \beta(\pi / 2)[1 \\
\left.+6 \cdot 16 \beta(0)(\beta(\pi / 2))^{-1}\right]^{-1}\left(4+16 L_{1} \beta(0)(\beta(\pi / 2))^{-1}\right)^{-3} \\
\geq 2^{-1}(\beta(0))^{-1} \beta(\pi / 2) \min \left\{1,\left(\pi^{2} / 16\right) \rho_{1} L_{1}^{-2}(\beta(0))^{-1}\right\} \\
\times 8^{-1} \beta(\pi / 2)(17 \cdot 6)^{-1}(\beta(0))^{-1} \beta(\pi / 2)\left(20 L_{1}\right)^{-3}(\beta(0))^{-3} \beta(\pi / 2)^{3} \\
=(\beta(0))^{-5} \beta(\pi / 2)^{6}\left(20 L_{1}\right)^{-3} \min \left\{1,\left(\pi^{2} / 16\right) \rho_{1} L_{1}^{-2}(\beta(0))^{-1}\right\}(17 \cdot 6)^{-1} 16^{-1} \\
=(\beta(0))^{-6}(\beta(\pi / 2))^{6} L_{1}^{-5} \rho_{1}\left(20^{3} \cdot 17 \cdot 16^{2} \cdot 6\right)^{-1} \pi^{2} \\
>(\beta(0))^{-6}(\beta(\pi / 2))^{6} L_{1}^{-5} \rho_{1}\left(20^{3} \cdot 16^{2}\right)^{-1}=400 \sigma_{1} .
\end{gathered}
$$

Thus (4.5) holds.

By (5.8), (5.6) and (5.1)

$$
\begin{aligned}
\sigma_{1} & =10^{-3} \beta(\pi / 2)\left[16 L_{1} \beta(0)(\beta(\pi / 2))^{-1}\right]^{-2}(8 \cdot 17)^{-1}(\beta(0))^{-4}(\beta(\pi / 2))^{3} L_{1}^{-3} \rho_{1} \\
& =\sigma_{0}(8 \cdot 17)^{-1}(\beta(0))^{-4}(\beta(\pi / 2))^{3} L_{1}^{-3} \rho_{1} \\
& <\sigma_{0}(8 \cdot 17)^{-1}(\beta(0))^{-4}(\beta(\pi / 2))^{3} L_{1}^{-1} 2 \beta(0) \leq \sigma_{0}(4 \cdot 17)^{-1} .
\end{aligned}
$$

Thus (4.4) is true and the assertion of Theorem 1.1 holds with $\sigma_{1}$ defined by (5.8).

Acknowledgements. The author is grateful to Victor Mizel for introducing the topic to him and for fruitful discussions and to the referee for helpful comments and suggestions. The part of this work was done while the author was visiting Carnegie Mellon University.

\section{REFERENCES}

[1] B. Dacorogna and C.E. Pfister, Wulff theorem and best constant in Sobolev inequality. J. Math. Pures Appl. 71 (1992) $97-118$.

[2] I. Fonseca, The Wulff theorem revisited. Proc. R. Soc. Lond. A 432 (1991) 125-145.

[3] J. Hannon, N.C. Bartelt, B.S. Swartzentruber, J.C. Hamilton and G.L. Kellogg, Step faceting at the (001) surface of boron doped silicon. Phys. Rev. Lett. 79 (1997) 4226-4229.

[4] J. Hannon, M. Marcus and V.J. Mizel, A variational problem modelling behavior of unorthodox silicon crystals. ESAIM: COCV 9 (2003) $145-149$.

[5] H.C. Jeng and E.D. Williams, Steps on surfaces: experiment and theory. Surface Science Reports 34 (1999) 175-294.

[6] W.W. Mullins, Theory of thermal grooving. J. Appl. Physics 28 (1957) 333-339. 